Efectos protectores renales de los antagonistas del receptor de angiotensina II en la diabetes de tipo 2

Aunque la inhibición de los efectos de la angiotensina II es beneficiosa en pacientes con nefropatía causada por la diabetes de tipo 1, todavía no se han publicado datos definitivos sobre la protección renal que puedan conferir los antagonistas del receptor de angiotensina II $\left(\mathrm{AT}_{1}\right)$ en pacientes con diabetes de tipo 2 . En un número reciente (20 de septiembre del 2001) de la revista New England Journal of Medicine se han publicado los resultados de tres ensayos clínicos que han abordado este problema.

En el ensayo clínico Irbesartan Diabetic Nephropathy Trial* se determinó si el uso de un antagonista del receptor $\mathrm{AT}_{1}$ o de un bloqueante de los canales del calcio podría proporcionar protección frente a la progresión de la nefropatía debida a la diabetes de tipo 2, independientemente de los efectos atribuibles a la reducción de la tensión arterial.

El estudio, aleatorio, prospectivo y doblemente enmascarado, se realizó en 210 centros clínicos. Los criterios de inclusión fueron: edad entre 30 y 70 años, diagnóstico de diabetes de tipo 2 e hipertensión, proteinuria $\geq 900 \mathrm{mg} / 24 \mathrm{~h}$ y creatinina sérica entre 1,0 y $3,0 \mathrm{mg} / \mathrm{dL}(88$ a $265 \mu \mathrm{mol} / \mathrm{L})$ en las mujeres, y entre 1,2 y $3,0 \mathrm{mg} / \mathrm{dL}(106$ a $265 \mu \mathrm{mol} / \mathrm{L})$ en los hombres. Los pacientes elegibles fueron asignados aleatoriamente al tratamiento con irbesartán (75 a $300 \mathrm{mg} /$ día), amlodipino (2,5 a $10 \mathrm{mg} /$ día) o placebo. Al menos 10 días antes de esta asignación, se suspendieron todos los tratamientos con inhibidores de la enzima de conversión de la angiotensina (IECA), antagonistas del receptor $\mathrm{AT}_{1}$ o bloqueantes de los canales del calcio. A partir de entonces, la hipertensión se controló con otros fármacos.

La proteinuria en $24 \mathrm{~h}$, las concentraciones séricas de creatinina y potasio, las variables cardiovasculares, la aparición de nefropatía terminal y la supervivencia se registraron cada 3 meses. La variable primaria fue la combinación de la duplicación de la concentración basal de creatinina sérica, la aparición de nefropatía terminal, definida como una concentración sérica de creatinina $\geq 6,0 \mathrm{mg} / \mathrm{dL}$ (530 $\mathrm{mmol} / \mathrm{L}$ ) o la necesidad de diálisis o trasplante renal, y la muerte por cualquier causa. La variable secundaria, cardiovascular, fue la combinación de la muerte por causas cardiovasculares, el infarto de miocardio no mortal, la insuficiencia cardíaca que necesitó hospitalización, las secuelas neurológicas permanentes ocasionadas por accidentes cerebrovasculares y las amputaciones del miembro inferior por encima del tobillo.

Entre el 21 de marzo de 1996 y el 25 de febrero de 1999 se aleatorizaron 1715 pacientes que fueron observados durante un promedio de 2,6 años. Las características demográficas, clínicas y de laboratorio iniciales eran similares en los tres grupos. En los pacientes tratados con irbesartán, el riesgo de presentar la variable primaria fue un $20 \%$ menor que en los tratados con placebo $(P=0,02)$ y un $23 \%$ menor que en los tratados con amlodipino $(P=0,006)$; entre estos dos últimos grupos no hubo diferencias significativas. El riesgo de duplicación de la creatinina sérica fue un $33 \%$ menor en el grupo tratado con irbesartán que en el tratado con placebo $(P=0,003)$ y un $37 \%$ menor que en el tratado con amlodipino $(P<$ $0,001)$. El riesgo relativo de nefropatía terminal en el grupo tratado con irbesartán fue un $23 \%$ menor que en cualquiera de los otros dos grupos $(P=0,07$ en ambas comparaciones). El aumento de la concentración sérica de creatinina en el grupo tratado con irbesartán fue un $24 \%$ más lento que en el tratado con placebo $(P=0,008)$ y un $21 \%$ más lento que en el tratado con amlodipino $(P=0,02)$. Con respecto a los desenlaces cardiovasculares, no hubo diferencias entre los grupos. La tensión arterial media en el grupo tratado con irbesartán no fue significativamente diferente de la observada en el grupo tratado con amlodipino y los análisis adicionales realizados revelaron que los mejores desenlaces renales de los pacientes tratados con irbesartán no se debían a diferencias en la tensión arterial media ni a desequilibrios en la distribución basal de las covariables.

Estos resultados indican que el irbesartán tiene efectos protectores renales en pacientes con diabetes de tipo 2 y nefropatía manifiesta y aminora significativamente la progresión de la glomerulopatía. Sus efectos beneficiosos, independientes de la reducción de la tensión arterial, se acompañaron de mínimos efectos adversos graves relacionados con el fármaco.

En el ensayo clínico Reduction of Endpoints in NIDDM with the Angiotensin II Antagonist Losartan $S t u d y^{\dagger}$, aleatorizado, doblemente enmascarado, controlado con placebo y con la participación de 250 centros de 28 países de Asia, Europa, Sudamérica, Centroamérica y Norteamérica, se investigó si el losartán, otro antagonista del receptor $\mathrm{AT}_{1}$, por sí solo o combinado con el tratamiento antihipertensor convencional, permitía prolongar el tiempo 
transcurrido hasta que se produjera la duplicación de la concentración sérica de creatinina, la aparición de nefropatía terminal o la muerte.

Los participantes fueron pacientes de 31 a 70 años diagnosticados de diabetes de tipo 2 y nefropatía. Los pacientes con hipertensión siguieron tomando sus medicaciones antihipertensoras, pero en caso de que estuvieran siendo tratados con IECA o antagonistas del receptor $\mathrm{AT}_{1}$ se les cambiaron estos fármacos por otros. Aleatoriamente, los pacientes fueron asignados al tratamiento con $50 \mathrm{mg}$ de losartán o placebo una vez al día, además del tratamiento antihipertensor convencional. La medida primaria de eficacia fue el tiempo transcurrido hasta la duplicación de la concentración sérica de creatinina, la aparición de nefropatía terminal o la muerte. Como medida secundaria se analizó la morbilidad y mortalidad por causas cardiovasculares (infarto de miocardio, accidente cerebrovascular, primera hospitalización por insuficiencia cardíaca o angina inestable, revascularización coronaria o periférica, o muerte por causas cardiovasculares). Otras medidas secundarias fueron la progresión de la nefropatía y los cambios de la proteinuria.

En total, se aleatorizaron 1513 pacientes que fueron observados durante una media de 3,4 años $(2,3$ a 4,6$)$. Las características basales eran similares en ambos grupos. Los pacientes que abandonaron el tratamiento fueron más numerosos entre los tratados con placebo $(53,5 \%)$ que entre los tratados con losartán $(46,5 \%)$ y eso se debió a acontecimientos adversos en el 21,7 y 17,2\% de los casos, respectivamente.

En comparación con el placebo, el losartán redujo el riesgo de presentar la variable primaria en un $16 \%(P=0,02)$ y esta disminución del riesgo apenas cambió $(15 \%)$ después del ajuste en función de la tensión arterial $(P=0,03)$. Además, el losartán redujo en un $25 \%(P=0,006)$ el riesgo de duplicación de la concentración sérica de creatinina, y en un $28 \%$ $(P=0,002)$ el riesgo de nefropatía terminal. En cambio, la tasa de mortalidad fue similar en ambos grupos. La variable compuesta que reflejaba la morbilidad y la mortalidad cardiovascular tampoco mostró diferencias entre los dos grupos, aunque la tasa de hospitalización por insuficiencia cardíaca fue significativamente menor con el losartán que con el placebo (reducción del riesgo del 32\%; $P=0,005$ ). La proteinuria disminuyó en un $35 \%$ con el losartán, en comparación con el placebo $(P=0,001)$.

Los resultados obtenidos indican que el losartán proporcionó importantes beneficios renales en pacientes con diabetes de tipo 2 y nefropatía y fue generalmente bien tolerado.

En el tercer estudio ${ }^{\ddagger}$, realizado en 96 centros de todo el mundo, aleatorizado y doblemente enmascarado, se investigó la eficacia del irbesartán para prevenir o retrasar la aparición de nefropatía diabética en pacientes hipertensos con diabetes de tipo 2 y microalbuminuria persistente. Además, también se investigó la dosis óptima de irbesartán para la protección renal.

Los criterios de inclusión fueron: edad entre 30 y 70 años, diagnóstico de hipertensión y diabetes de tipo 2, microalbuminuria (20 a $200 \mu \mathrm{g} / \mathrm{min}$ ) persistente y creatinina sérica $\leq 1,5 \mathrm{mg} / \mathrm{dL}$ (133 $\mu \mathrm{mol} / \mathrm{L})$ en los hombres $\mathrm{y} \leq 1,1 \mathrm{mg} / \mathrm{dL}(97 \mu \mathrm{mol} /$ L) en las mujeres. Tras una fase de preinclusión en la que se retiraron todos los tratamientos antihipertensores y se sustituyeron por placebo, se procedió a la asignación aleatoria de 590 pacientes al tratamiento, una vez al día, con 150 o 300 mg de irbesartán, o placebo. Además, se permitió el uso de otros antihipertensores (diuréticos, $\beta$-bloqueantes, bloqueantes de los canales del calcio, excepto las dihidropiridinas, y $\alpha$-bloqueantes), siempre que no fueran IECA. Los pacientes fueron observados durante una mediana de 2 años y los exámenes se efectuaron en el momento de la aleatorización, a las 2 y a las 4 semanas, y a los 3, 6, 12, 18, 22 y 24 meses. La medida primaria de eficacia fue el tiempo transcurrido entre la consulta inicial y la detección por primera vez de nefropatía manifiesta, definida como una albuminuria $>200 \mu \mathrm{g} / \mathrm{min}$ y al menos un $30 \%$ mayor que al principio del estudio. Como variables secundarias se incluyeron los cambios de la albuminuria y del aclaramiento de creatinina, y la restauración de la normoalbuminuria $(<20 \mu \mathrm{g} / \mathrm{min})$ al final del estudio.

Las características demográficas, clínicas y bioquímicas iniciales eran similares en los tres grupos. A lo largo de los 2 años apareció nefropatía en 30 de los 201 pacientes $(14,9 \%)$ tratados con placebo, en 19 de los 195 (9,7\%) tratados con $150 \mathrm{mg}$ de irbesartán y en 10 de los 194 (5,2\%) tratados con 300 mg de irbesartán. Las curvas de Kaplan-Meier de los grupos tratados con placebo y con $300 \mathrm{mg}$ de irbesartán empezaron a separarse en la consulta realizada a los 3 meses, y siguieron divergiendo hasta el final del estudio. La razón no ajustada de riesgo instantáneo de nefropatía diabética fue de 0,61 , con un intervalo de confianza del 95\% (IC95) de 0,34 a $1,08(P=0,08)$, en el grupo tratado con $150 \mathrm{mg}$ de irbesartán y de 0,30 (IC95: 0,14 a 0,61; $P<0,001$ ) en el tratado con $300 \mathrm{mg}$. Tras el ajuste en función de la microalbuminuria basal y de la tensión arterial alcanzada a lo largo del estudio, las cifras correspondientes fueron de 0,56 (IC95: 0,31 a 0,99; $P=0,05$ ) y 0,32 (IC95: 0,15 a 0,65; $P<0,001$ ), respectivamente.

Además, el irbesartán redujo la excreción urinaria de albúmina a lo largo del estudio: en un $24 \%$ (IC95: 19 a 29\%) con $150 \mathrm{mg}$ y en un 38\% (IC95: 32 a $40 \%$ ) con $300 \mathrm{mg}$, frente a tan solo un $2 \%$ (IC95: -7 a $5 \%)$ con el placebo $(P<0,001$ para la comparación entre el placebo y los dos grupos de irbesar- 
tán combinados). Dicha reducción fue significativamente menor con la dosis de $150 \mathrm{mg}$ que con la de $300 \mathrm{mg}(P<0,001)$.

La restauración de la normoalbuminuria al final del estudio también fue más frecuente en los grupos tratados con $300 \mathrm{mg}$ (34\%; IC95: 26 a $40 \%$ ) y $150 \mathrm{mg}$ de irbesartán (24\%; IC95: 18 a $30 \%$ ) que en el tratado con placebo (21\%; IC95: 15 a $26 \%$; $P=0,006$ para la comparación entre el placebo y los dos grupos de irbesartán combinados).

Durante el tratamiento y las 2 semanas siguientes, se registraron acontecimientos adversos graves en el $22,8 \%$ de los pacientes tratados con placebo y en el $15,4 \%$ de los dos grupos tratados con irbesartán $(P=0,02)$. Los accidentes cardiovasculares no mortales también fueron ligeramente más frecuentes con el placebo $(8,7 \%)$ que con el irbesartán $(4,5 \%$ con $300 \mathrm{mg})$, y lo mismo ocurrió con los abandonos del tratamiento $(18,9 \%$ con el placebo y $14,9 \%$ en el conjunto de los dos grupos tratados con irbesartán). Estas dos últimas diferencias no fueron estadísticamente significativas.

Este estudio revela que el irbesartán reduce de forma significativa el ritmo de progresión hacia la albuminuria clínicamente manifiesta en pacientes con diabetes de tipo 2. Además, el retorno a la normoalbuminuria fue significativamente más frecuente en los pacientes tratados con $300 \mathrm{mg}$ de irbesartán. Estos beneficios parecían ser independientes de la tensión arterial sistémica, dado que la tensión arterial inmediatamente antes de la siguiente toma solo fue ligeramente menor en los grupos tratados con irbesartán que en el tratado con placebo.

Los tres estudios coinciden en señalar que los antagonistas del receptor $\mathrm{AT}_{1}$ reducen el riesgo de nefropatía en pacientes con diabetes de tipo 2. Estos datos vienen a unirse a la abundante literatura que demuestra que los IECA aminoran la progresión de la nefropatía proteinúrica, incluida la de los pacientes con diabetes de tipo 1, de forma más eficaz que muchos otros medicamentos. A esto hay que añadir también los resultados de otro estudio a gran escala (el Heart Outcomes Prevention Evaluation Study, conocido como MICRO-HOPE) que reveló que los IECA confieren protección frente a los accidentes cardiovasculares y atenúan el aumento de la proteinuria en pacientes con diabetes de tipo 2. Así pues, en comparación con otros fármacos, los bloqueantes del sistema renina-angiotensina-aldosterona probablemente produzcan una mayor disminución de la presión capilar glomerular o de las acciones fibroproliferativas de la aldosterona y la angiotensina. También hay datos que indican que los IECA proporcionarían una buena relación costo-efectividad si se prescribieran a todos los pacientes de edad media con diabetes de tipo 2, independientemente de su función renal inicial. Sin embargo, debido al mayor costo de los antagonistas del receptor $\mathrm{AT}_{1}$, es posible que esta conclusión no sea aplicable a esta clase de fármacos. ("Lewis EJ, Hunsicker LG, Clarke WR, Berl T, Pohl MA, Lewis JB, et al. Renoprotective effect of the angiotensin-receptor antagonist irbesartan in patients with nephropathy due to type 2 diabetes. N Engl J Med 2001;345: 851-860. 'Brenner BM, Cooper ME, de Zeeuw D, Keane WF, Mitch WE, Parving Hans-Henrik, et al. Effects of losartan on renal and cardiovascular outcomes in patients with type 2 diabetes and nephropathy. $\mathrm{N}$ Engl J Med 2001;345: 861-869. ‡Parving H-H, Lehnert H, Brochner-Mortensen J, Gomis R, Andersen S, Arner $P$. The effect of irbesartan on the development of diabetic nephropathy in patients with type 2 diabetes. N Engl J Med 2001;345: 870-878)

\section{Universalización de las pruebas de detección de la diabetes en mayores de 45 años}

La ADA (American Diabetes Association) propuso en 1997 que todos los individuos de más de 45 años se sometan cada 3 años a pruebas de detección de la diabetes mediante la determinación de la glucemia en ayunas. Tal política tendría gran impacto en los recursos del Servicio Nacional de Salud del Reino Unido, donde sigue el debate acerca de esta estrategia. Por consiguiente, los autores del presente informe decidieron investigar la factibilidad de esta recomendación de la ADA en el Reino Unido, para lo cual seleccionaron una consulta de medicina general con una población mayoritariamente blanca y con riesgo relativamente bajo. Asimismo, se investigaron los factores de riesgo de los diabéticos diagnosticados de esta forma, con el fin de determinar si se trataba de una población de alto riesgo no identificada hasta entonces.

Se realizó un estudio de población transversal en el que se invitó a participar a todos los individuos adscritos a la consulta seleccionada que tuvieran más de 45 años y no estuvieran diagnosticados de diabetes. Además de una determinación de la glucemia en ayunas se les interrogó acerca de los antecedentes de hipertensión y tratamientos antihipertensores, consumo de tabaco y antecedentes familiares de diabetes, se midió la tensión arterial, la talla y el peso, y se calculó el índice de masa corporal.

A los pacientes con glucemia en ayunas $\geq 6,1$ $\mathrm{mmol} / \mathrm{L}$ se les envió una carta solicitándoles que volvieran a la consulta para la realización de pruebas diagnósticas. A aquellos con glucemias de 6,1$6,9 \mathrm{mmol} / \mathrm{L}$ se les realizó una prueba de tolerancia a la glucosa oral (75 g) y a aquellos con glucemias $\geq 7 \mathrm{mmol} / \mathrm{L}$ también se les repitió la determinación de la glucemia en ayunas. Los pacientes fueron clasificados según los criterios diagnósticos de la ADA 
(tolerancia normal a la glucosa, deterioro de la glucemia en ayunas o diabetes) y de la Organización Mundial de la Salud (tolerancia normal a la glucosa, deterioro de la tolerancia a la glucosa, deterioro de la glucemia en ayunas o diabetes). A los pacientes que acudieron a una segunda consulta también se les realizó un lipidograma completo (colesterol total, colesterol de las lipoproteínas de alta densidad y triglicéridos) y se calculó su riesgo de cardiopatía coronaria a 10 años.

Entre los 2596 individuos con más de 45 años había 115 ya diagnosticados de diabetes. De los otros 2481,876 aceptaron participar en el estudio y fueron sometidos a la determinación de la glucemia en ayunas. No se observaron diferencias estadísticamente significativas entre las características demográficas de los participantes y de los no participantes. Se identificaron 60 casos con glucemia $\geq 6,1 \mathrm{mmol} / \mathrm{L} \mathrm{y}$ 45 de ellos acudieron a la segunda consulta.

Excepto uno, todos los pacientes diagnosticados de diabetes, deterioro de la glucemia en ayunas o deterioro de la tolerancia a la glucosa tenían otros factores de riesgo además de la edad. En la población examinada hubo 495 pacientes con otros factores de riesgo (hipertensión, antecedentes de diabetes en un familiar en primer grado o índice de masa corporal $>27 \mathrm{~kg} / \mathrm{m}^{2}$ ). Revisando los registros de la consulta, se identificó un total de 1027 individuos de más de 45 años con uno o más factores de riesgo adicionales, aunque este número probablemente sea inferior al real, dado que había pocas historias que mencionaran los antecedentes familiares de diabetes.

Utilizando el valor de $6,1 \mathrm{mmol} / \mathrm{L}$, la prevalencia de diabetes no diagnosticada anteriormente fue del 1,7\% (intervalo de confianza del 95\%: 0,7 a $2,8 \%$ ) en la totalidad de la población examinada, del $2,8 \%(1,6$ a $4,7 \%)$ en pacientes con otros factores de riesgo, además de la edad, y del $0,2 \%$ ( 0 a 1,4\%) en pacientes sin otros factores de riesgo. En la población examinada hubo 312 pacientes con un factor de riesgo adicional, 2 de los cuales fueron diagnosticados de diabetes (prevalencia del 0,6\%; 0,1 a 2,3\%); 159 con dos, 7 de los cuales fueron diagnosticados de diabetes (prevalencia del $4,4 \% ; 1,8$ a $8,9 \%$ ), y 24 con tres, 4 de los cuales fueron diagnosticados de diabetes (prevalencia del 16,7\%; 4,7 a 37\%).

Se calculó que serían necesarias 4 horas semanales durante un año para realizar el procedimiento investigado en la totalidad de la población de más de 45 años adscrita a la consulta y que cerca de la mitad de ese tiempo correspondería a pacientes con otros factores de riesgo además de la edad. Más del $80 \%$ de los pacientes diagnosticados de diabetes de novo tenían un riesgo de cardiopatía coronaria a 10 años > 15\%, el 73\% (45 a 92\%) eran hipertensos y el $73 \%$ (45 a $92 \%)$ tenía concentraciones de colesterol $>5 \mathrm{mmol} / \mathrm{L}$.
Los autores concluyen que la detección de la diabetes mediante la determinación de la glucemia en ayunas es factible en las consultas de medicina general y que permitiría identificar a un grupo de pacientes con alto riesgo de enfermedad cardiovascular. Sin embargo, esto requeriría una considerable inversión de tiempo del personal y, como el número de casos identificados basándose únicamente en la edad es bajo, las pruebas de detección deberían reservarse para los pacientes con múltiples factores de riesgo de diabetes. (Lawrence JM, Bennett P, Young A, Robinson AM. Screening for diabetes in general practice: cross sectional population study. BMJ 2001;323:548-551)

\section{Insulina en aerosol}

Todos los pacientes con diabetes de tipo 1, y muchos con diabetes de tipo 2, necesitan tratamiento con insulina. La necesidad de inyectarse, en algunos casos varias veces al día, dificulta el cumplimiento del tratamiento. Varios autores han investigado la posibilidad de administrar la insulina en aerosol, con el fin de evitar las molestias ocasionadas por las inyecciones. En este artículo se revisan los conocimientos actuales sobre este tema.

Se ha investigado la posibilidad de desarrollar formulaciones en aerosol que pudieran administrarse por vía intranasal o intrapulmonar. La vía intranasal tiene la ventaja de su fácil acceso, pero también el inconveniente de que la superficie de absorción es pequeña (unos $150 \mathrm{~cm}^{2}$, frente a los $75 \mathrm{~m}^{2}$ del pulmón) y de que el fármaco se tiene que absorber rápidamente, en unos 15 a 20 minutos, antes de que sea eliminado por el rápido mecanismo de aclaramiento mucociliar nasal. Varios investigadores han registrado importantes reducciones de la glucemia con la administración intranasal de aerosoles de insulina, pero solo cuando se les añaden agentes tensioactivos, que pueden producir irritación y congestión nasal. Por consiguiente, esta no parece ser la vía de elección para la administración de aerosoles de insulina.

Para una administración óptima de fármacos en aerosol por vía intrapulmonar es necesario que el aerosol se deposite en los alveolos, y la mejor forma de conseguirlo es por inhalación oral. Sin embargo, la inhalación oral también tiene inconvenientes: las partículas $>5 \mu \mathrm{m}$ chocan con la parte posterior de la cavidad bucal y no penetran en las vías respiratorias inferiores. A las partículas de aerosol con grandes velocidades, como las generadas por propulsores, $\mathrm{y}$ a las inhaladas con grandes flujos inspiratorios $(>30$ $\mathrm{L} / \mathrm{min}$ ) les ocurre lo mismo, lo cual podría explicar que en varios estudios esta vía de administración se haya mostrado poco eficaz para reducir la glucemia. 
En algunos estudios, solo el 10\% del fármaco nebulizado se ha depositado en los pulmones. En la actualidad está demostrado que el tamaño de las partículas del aerosol, su velocidad y el flujo inspiratorio son los principales factores que determinan la llegada de los aerosoles a los pulmones.

En individuos sanos, el tiempo transcurrido hasta la consecución de la máxima concentración de insulina tras su administración intrapulmonar ha oscilado entre 15 a 20 y 50 a 60 minutos, frente a los 85 a 144 minutos registrados tras la administración subcutánea. Esto indica que la insulina se absorbe con mayor rapidez en los pulmones de los individuos sanos, pero estudios similares realizados en pacientes con diabetes de tipo 2 no han mostrado diferencias significativas entre ambas vías de administración. Se desconoce la causa de esta diferencia entre los individuos sanos y los pacientes con diabetes de tipo 2, pero podría deberse a diferencias entre la absorción de la insulina regular porcina utilizada en los estudios de pacientes diabéticos y la insulina recombinante humana utilizada en los estudios de individuos sanos, a que la enfermedad altere la absorción de la insulina a través del epitelio pulmonar, o simplemente a la variabilidad interindividual.

La biodisponibilidad media de las dosis inhaladas de aerosoles de insulina es de aproximadamente un $20 \%$, en comparación con las dosis administradas por vía subcutánea. Estos datos se refieren a la insulina nebulizada y no se sabe cómo podrían verse afectados por cambios de la formulación, tales como la sustitución de la insulina líquida por insulina en polvo. También hay que tener en cuenta que las determinaciones han sido hechas en no fumadores y que el tabaco parece tener un importante efecto sobre la biodisponibilidad de los aerosoles nebulizados de insulina, aumentándola. La menor biodisponibilidad de la insulina en aerosol en comparación con la insulina subcutánea puede incrementar el costo del tratamiento.

El autor no tiene conocimiento de que se hayan producido casos de toxicidad tras la administración a corto plazo de insulina por vía inhalatoria. En una revisión de los estudios publicados hasta 1996 acerca de tratamientos a más largo plazo tampoco se observaron efectos pulmonares adversos y en tres estudios de fase II en los que el tratamiento se administró durante 3 meses no se observaron cambios en las pruebas funcionales pulmonares. No obstante, son necesarios nuevos estudios para investigar la posible aparición de inmunogenicidad y toxicidad tras la exposición pulmonar prolongada.

Antes de que la insulina en aerosol se transforme en una alternativa factible a la insulina subcutánea serán necesarios nuevos estudios, en particular sobre el sistema de administración. Los sistemas actuales tienen el inconveniente de que la mayor parte de la dosis se queda en el dispositivo y no llega a los pulmones. También se están investigando nuevas formulaciones de aerosoles de insulina, la mayoría solubilizadas o en polvo. Otro aspecto que necesita ser investigado es el de la influencia de la función pulmonar basal del paciente sobre el depósito de los aerosoles de insulina en los pulmones.

En resumen, con la insulina, como con otros péptidos y proteínas, el éxito de los tratamientos con aerosoles depende de una correcta dosificación y formulación del fármaco, así como de la creación de dispositivos óptimos que posibiliten la llegada de la máxima cantidad de fármaco a los alveolos pulmonares. (Laube BL. Treating diabetes with aerosolized insulin. Chest 2001:120(Supl.):99S-106S)

\section{Efectos del ejercicio sobre el control de la glucemia y la masa corporal en la diabetes de tipo 2. Metaanálisis de los ensayos clínicos controlados}

El ejercicio, la dieta y la farmacoterapia constituyen las bases del tratamiento de la diabetes. Aunque se han realizado numerosos estudios pequeños sobre los efectos del ejercicio en pacientes con diabetes de tipo 2 , sus resultados han sido contradictorios y no ha habido estudios a gran escala con suficiente potencia estadística. En algunos estudios el ejercicio ha reducido la hemoglobina glucosilada $\left(\mathrm{HbA}_{1 \mathrm{c}}\right)$, pero en otros no. Dado que los metaanálisis son especialmente útiles cuando existen estudios con un pequeño número de pacientes y resultados contradictorios, los autores de este artículo realizaron una revisión sistemática de los efectos de los programas de ejercicio sobre el control de la glucemia, representado por la concentración de $\mathrm{HbA}_{1 c}$ y la masa corporal, medida como peso corporal $(\mathrm{kg})$ o índice de masa corporal $\left(\mathrm{kg} / \mathrm{m}^{2}\right)$.

Para identificar los estudios pertinentes se efectuó una búsqueda en diferentes bases de datos, entre ellas MEDLINE 1966-2000, EMBASE 19802000 y el Registro Cochrane de Ensayos Clínicos Controlados. Además se revisaron manualmente las listas de referencias bibliográficas de los principales libros de texto, de los artículos de revisión y de todos los artículos identificados en la búsqueda informatizada. Se incluyeron los artículos publicados en lengua no inglesa y se consultó con los expertos sobre la posible existencia de literatura no publicada.

En el análisis solo se incluyeron los estudios en los que el programa de ejercicio tuviera una duración mínima de 8 semanas. Los programas tenían que describir el tipo, frecuencia, intensidad y duración del ejercicio y este tenía que ser verificado por supervisión directa o mediante diarios de ejercicio. Se excluyeron aquellos estudios en los que solo se recomendó 
aumentar la actividad física, sin más especificaciones, y también aquellos en los que el ejercicio se acompañó de intervenciones farmacoterapéuticas. La intensidad del ejercicio se expresó en equivalentes metabólicos (MET), y su volumen (gasto energético total durante el ejercicio) en MET-hora. La calidad metodológica de los estudios analizados se clasificó con una escala validada de 5 puntos.

Las búsquedas informatizadas proporcionaron aproximadamente 2700 artículos, 1487 de los cuales estaban clasificados como ensayos clínicos controlados. Finalmente, en el análisis se incluyeron solo 14 estudios, con un total de 504 participantes. Su calidad fue moderada o baja, con una puntuación media ( \pm desviación estándar) de 1,6 \pm 0,5, de un total de 5 puntos posibles. Once estudios fueron aleatorizados; los otros 3 fueron simplemente ensayos clínicos controlados; ninguno tuvo doble enmascaramiento.

El cumplimiento del programa de ejercicios fue relativamente alto. En términos generales, las intervenciones prescribieron tres sesiones semanales, de $53 \pm 17$ minutos, durante $18 \pm 15$ semanas. En 12 estudios se utilizaron ejercicios aeróbicos, de intensidad moderada, generalmente consistentes en caminar o andar en bicicleta. En los otros dos se utilizaron ejercicios de resistencia.

Las concentraciones de $\mathrm{HbA}_{1 \mathrm{c}}$ después de la intervención fueron significativamente menores en los grupos que realizaron ejercicio que en los grupos de control $(7,65 \%$ frente a $8,31 \%$; diferencia media de $-0,66 \% ; P<0,001)$. Cuando el ejercicio se combinó con dieta, la diferencia $(-0,76 \% ; P=0,008)$ fue similar a la obtenida solo con el ejercicio. En cambio, no se observaron diferencias significativas entre los grupos con respecto a la masa corporal (diferencia media estandarizada de 0,$06 ; P=0,60$ ).

Cinco modelos diferentes de análisis de metarregresión revelaron que la diferencia media en el peso corporal tras la intervención no predecía la diferencia media en las concentraciones de $\mathrm{HbA}_{1 \mathrm{c}}$ (modelo 1); que esto seguía siendo así incluso después del ajuste en función de los valores basales (modelo 2); que la intensidad del ejercicio tampoco se asociaba a la diferencia media en las concentraciones de $\mathrm{HbA}_{1 \mathrm{c}}$ (modelo 3); que lo mismo ocurría con el volumen de ejercicio (modelo 4), y que estas tres variables (peso corporal e intensidad y volumen del ejercicio), incorporadas simultáneamente como variables independientes, solo explicaban una fracción no significativa $(8 \%)$ de la varianza de las concentraciones de $\mathrm{HbA}_{1 \mathrm{c}}$ (modelo 5).

Aunque los diferentes estudios clínicos realizados hasta ahora acerca de los efectos del ejercicio en pacientes con diabetes de tipo 2 han proporcionado resultados contradictorios, en este metaanálisis se detectó una reducción de la $\mathrm{Hb}_{1 \mathrm{c}}$ de aproxima- damente $0,66 \%$, cifra que podría ser clínicamente importante y asociarse a una reducción significativa de las complicaciones de la diabetes. El hecho de que no hubiera diferencias entre los grupos con respecto a la masa corporal también es importante e indica que el ejercicio es beneficioso por sí mismo, y no a consecuencia del adelgazamiento. Las investigaciones futuras sobre este tema deberían incluir intervenciones más prolongadas y una mejor cuantificación de los cambios de la composición corporal. Mientras tanto, los resultados de este metaanálisis respaldan una vez más la idea de que el ejercicio es un componente fundamental del tratamiento de la diabetes. (Boulé NG, Haddad E, Kenny GP, Wells GA, Sigal RJ. Effects of exercise on glycemic control and body mass in type 2 diabetes mellitus. A meta-analysis of controlled clinical trials. JAMA 2001;286: 1218-1227)

\section{Prevalencia de los síntomas gastrointestinales en la diabetes}

Los síntomas gastrointestinales (SGI) son frecuentes en pacientes diabéticos y generalmente se atribuyen a la neuropatía neurovegetativa. Aunque los SGI afectan negativamente a la calidad de vida y constituyen una causa considerable de morbilidad, los datos epidemiológicos sobre su prevalencia y determinantes son contradictorios y los estudios realizados hasta ahora tienen importantes deficiencias metodológicas. Los autores de este estudio realizaron una encuesta epidemiológica para comprobar si los SGI son más prevalentes en los pacientes con diabetes que en los individuos sin esta enfermedad y si se asocian al mal control de la glucemia.

A partir del censo electoral, se seleccionaron aleatoriamente 15000 individuos residentes en una zona de Australia cuya población tiene características demográficas similares a las del resto del país. La encuesta se realizó entre el 1 de agosto y el 15 de septiembre de 1999. Por vía postal, se le envió a cada individuo seleccionado un cuestionario con 16 preguntas sobre la frecuencia de los SGI en los 3 meses anteriores. Los 16 síntomas se agruparon en 5 complejos sintomáticos: síntomas esofágicos, síntomas de dismotilidad de las vías superiores, cualquier síntoma intestinal, síntomas diarreicos y síntomas de estreñimiento. Se consideraron diabéticos los individuos que contestaron afirmativamente a la pregunta " ¿Alguna vez ha sido diagnosticado de diabetes por un médico?" y se les pidió que proporcionaran información sobre la duración y el tratamiento de la diabetes (solo con insulina, con insulina e hipoglucemiantes orales, solo con hipoglucemiantes orales o solo con dieta) y que puntuaran su control de la glucemia en una escala de Likert de 5 puntos: muy bueno, bueno, intermedio, malo o muy malo. Para el 
análisis, las dos primeras categorías se combinaron en una sola ("bueno"), y las dos últimas en otra ("malo"). La diabetes se consideró de tipo 1 cuando el diagnóstico se estableció antes de los 30 años y el paciente estaba siendo tratado con insulina, y de tipo 2 en todos los demás casos. Los casos de diabetes exclusivamente gestacional fueron excluidos.

La prevalencia de SGI en la población general se estimó en un 2 a 15\% y se consideró clínicamente importante un aumento del $10 \%$ en los pacientes diabéticos. Con estas cifras, se calculó que, para obtener una potencia estadística de 0,9 con un nivel de significación de 0,05 serían necesarios 120 pacientes con diabetes en caso de que la prevalencia fuera del $2 \%$, y 335 en caso de que fuera del $15 \%$. La asociación entre la diabetes y la proporción de pacientes con y sin determinado síntoma o complejo sintomático se analizó mediante regresión logística con ajustes en función de la edad y el sexo. Los resultados se expresaron como razones de los productos cruzados (odds ratios: OR), con sus intervalos de confianza del 95\% (IC95).

De los 15000 cuestionarios enviados, 429 fueron devueltos por dirección desconocida o incorrecta y 99 por muerte reciente o ausencia del destinatario. De los 14472 restantes, se completaron y devolvieron 8657 (60\%). El 5,4\% de los individuos (472) refirieron ser diabéticos, pero 49 eran mujeres que solo habían padecido diabetes gestacional. Por consiguiente, en el análisis se incluyeron 423 pacientes con diabetes $(4,9 \%)$. La duración media (intervalo intercuartílico: II) de la enfermedad fue de 5 años $(2,2$ a 11,0$)$. La mayoría de los pacientes $(94,8 \%)$ tenían diabetes de tipo 2 y estaban siendo tratados con hipoglucemiantes orales.

El número medio de SGI por individuo fue mayor en los pacientes (1,3; II: 0 a 2$)$ que en los controles (0,96; II: 0 a $1 ; P<0,001)$. Todos los SGI y los cinco complejos sintomáticos fueron significativamente más prevalentes en los pacientes que en los controles, incluso tras el ajuste por sexo y edad. Las asociaciones más fuertes correspondieron a la incontinencia fecal (OR: 2,74; IC95: 1,40 a 5,37), la disfagia (OR: 2,71; IC95: 1,69 a 4,36) y los vómitos (OR: 2,51; IC95: 1,12 a 5,66).

Tras los ajustes por edad y sexo, el mal control de la glucemia se asoció a la prevalencia de los 5 complejos sintomáticos y de 12 de los 16 síntomas investigados; cuanto peor el control, mayor la prevalencia. Los pacientes con mal control de la glucemia también presentaron un número medio de síntomas (3,49; II: 0 a 6$)$ mayor que los pacientes con control intermedio (1,38; II: 0 a $2 ; P<0,001)$ o bueno $(0,89 ;$ II: 0 a $1 ; P<0,001)$.

La duración de la glucemia no se asoció de forma significativa a la prevalencia de los SGI. Los pacientes con diabetes de tipo 1 tuvieron tendencia a presentar menos SGI que aquellos con diabetes de tipo 2, pero solo el complejo sintomático "intestinal" fue significativamente más frecuente en estos últimos (OR: 8,33; IC95: 1,39 a 50,0).

Se efectuaron tres análisis para explorar las asociaciones entre los tipos de tratamiento de la diabetes y la prevalencia de los SGI. En el primero (pacientes tratados con insulina frente a los no tratados con insulina) no se observaron diferencias con respecto a ningún síntoma ni complejo sintomático. En el segundo (pacientes tratados con hipoglucemiantes orales frente a los no tratados con estos fármacos), solo el bloqueo anal fue significativamente menos frecuente en los primeros (OR: 0,38; IC95: 0,16 a 0,88). En el tercero (pacientes tratados únicamente con dieta frente a los tratados con hipoglucemiantes, insulina o ambos), la plenitud posprandial (OR: 2,29; IC95: 1,10 a 4,77), el bloqueo anal (OR: 2,84; IC95: 1,29 a 6,24) y el complejo sintomático "estreñimiento" (OR: 2,09; IC95: 1,07 a 4,08) fueron significativamente más frecuentes en los pacientes tratados solo con dieta.

Al contrario de los estudios anteriores sobre este problema, este se basó en la población e incluyó un grupo de control adecuado y una población representativa de pacientes diabéticos de todas las edades y grados de gravedad de la enfermedad. Los resultados obtenidos revelan que los SGI son más prevalentes en los pacientes diabéticos que en los individuos sin esta enfermedad, e indican que este efecto puede deberse, al menos en parte, al mal control de la glucemia, pero no parece estar relacionado con la duración de la diabetes ni el tipo de tratamiento. (Bytzer P, Talley NJ, Leemon M, Young LJ, Jones MP, Horowitz M. Prevalence of Gastrointestinal symptoms associated with diabetes mellitus: a population-based survey of 15000 adults. Arch Intern Med 2001;161:1989-1996)

\section{La diabetes y el riesgo de enfermedad de Alzheimer y demencia con accidente cerebrovascular en una cohorte multiétnica}

La demencia y la diabetes sacarina son dos de las enfermedades más prevalentes en los ancianos, ambas con cifras superiores al $10 \%$ en los mayores de 65 años. La asociación entre la diabetes y la demencia vascular puede depender de la presencia de enfermedad cerebrovascular subclínica o de accidentes cerebrovasculares (ACV) francos y puede ser mediada por factores de riesgo tradicionales de enfermedad cardiovascular, como la hiperlipidemia y la hipertensión. En cambio, la relación entre la diabetes y la enfermedad de Alzheimer (EA) no es tan clara. Los estudios sobre la asociación entre la diabetes y la EA han proporcionado resultados contradictorios. El hecho de que se detecte o no una relación entre la diabetes y la demencia, específicamente 
la EA y la demencia vascular, puede depender de los criterios utilizados para definir la demencia vascular y de la exactitud de los procedimientos diagnósticos empleados. A pesar de que la demencia y la diabetes son más frecuentes en negros e hispanoamericanos, ninguno de los estudios anteriores sobre la relación entre ambas enfermedades incluyó a un gran número de individuos de estos grupos.

El objetivo de este estudio consistió en esclarecer la relación entre la diabetes y los diferentes tipos de demencia. Como la prevalencia de la diabetes es mayor en los negros y en los hispanoamericanos, también se investigó si esta diferencia podría explicar el mayor riesgo de demencia registrado en estos grupos.

El estudio se realizó en miembros de la cohorte del Washington Heights-Inwood Columbia Aging Project, constituida por individuos de 65 años o más, seleccionados aleatoriamente entre los residentes de un área definida del norte de Manhattan, Nueva York. A cada individuo se le realizó una entrevista estructurada, una anamnesis, una exploración física y neurológica y una serie de pruebas neuropsicológicas. Los participantes fueron reclutados entre 1991 y 1996 y fueron examinados anualmente durante una media de 4,3 años, entre 1992 y 1997. En el análisis se incluyeron aquellos que completaron al menos 1 año de seguimiento. De los 2126 individuos examinados inicialmente, se excluyeron 327 que ya padecían demencia al comenzar el estudio y otros 537 que no acudieron a las consultas de seguimiento. La muestra analizada consistió en 1262 individuos.

El diagnóstico de demencia se basó en los criterios DSM-IV y requirió la presencia de déficit cognitivos en las pruebas neuropsicológicas y signos de deterioro del funcionamiento social o laboral (Puntuación Clínica de la Demencia >0,5), y el de EA en los criterios NINCDS-ADRDA. El diagnóstico de demencia asociada a ACV se estableció en todos los individuos con demencia en los que se consideró que su principal causa eran los ACV. El diagnóstico de deterioro cognitivo no demencial (DCND) se basó en la presencia de trastornos de la memoria en las pruebas neuropsicológicas, sin deterioro del funcionamiento social o laboral (Puntuación Clínica de la Demencia < 0,5). El diagnóstico de la diabetes se basó en el uso de insulina o hipoglucemiantes orales o en la presencia de antecedentes clínicos de diabetes.

Se comparó la prevalencia de la diabetes y de otras covariables en individuos con y sin EA y en individuos con y sin demencia asociada a ACV. Las variables continuas se compararon mediante análisis de varianza, y las categóricas mediante la prueba de la $\chi^{2}$. En los análisis multivariados se utilizó el modelo de riesgos instantáneos proporcionales de Cox. El riesgo poblacional de demencia atribuible a la diabetes se calculó en los diferentes grupos étnicos con la fórmula $\mathrm{PAR}=\operatorname{Pr}(\mathrm{Hr}-1)$ / $1+\operatorname{Pr}(\mathrm{Hr}-1)$, donde PAR es el riesgo poblacional atribuible, HR la razón de riesgos instantáneos ajustada obtenida con los modelos multivariados, y $\mathrm{Pr}$ la prevalencia de la diabetes en cada grupo étnico.

La media de edad de la cohorte fue de 75,6 años (desviación estándar: 5,9). El 68,9\% de los participantes eran mujeres, el $45 \%$ hispanoamericanos y el $32 \%$ negros. La prevalencia de la diabetes fue del $9,6 \%$ en los blancos, del $21,2 \%$ en los negros y del $24,1 \%$ en los hispanoamericanos. Se registraron 213 nuevos casos de demencia: 157 (74\%) debidos a la EA, $36(17 \%)$ a ACV y $20(9 \%)$ a otras causas. La incidencia de demencia fue de 1,4/1 000 personasaño en los blancos, de 2,4 en los negros y de 2,3 en los hispanoamericanos.

Las HR relacionadas con la diabetes, en comparación con la ausencia de diabetes, fueron de 1,3 (IC95\%: 0,8 a 1,9) para la EA, de 3,4 (IC95\%: 1,7 a 6,9) para la demencia asociada a ACV, de 1,3 (IC95\%: 0,8 a 1,9) para el DCND sin ACV, y de 1,6 (IC95\%: 0,6 a 4,4) para el DCND con ACV. Para la demencia asociada a ACV, el riesgo poblacional atribuible a la diabetes fue del 36\% (IC95\%: 33 a $37 \%$ ) en los hispanoamericanos, del 33\% (IC95\%: 31 a $36 \%$ ) en los negros no hispanoamericanos y del 17\% (IC95\%: 13 a 22\%) en los blancos no hispanoamericanos. Como los individuos con DCND corren mayor riesgo de padecer EA que aquellos sin DCND, también se realizó un análisis de la relación entre la diabetes y la variable combinada EA más DCND sin ACV. Para esta variable, la HR relacionada con la diabetes, en comparación con la ausencia de diabetes, fue de 1,6 (IC95\%: 1,2 a 2,1).

La débil asociación, no significativa, entre la diabetes y la EA podría explicarse por una insuficiente potencia estadística del estudio para detectarla. A favor de esta explicación se encuentra el hecho de que la combinación de la EA con el DCND sin ACV mostrara una significativa asociación con la diabetes. Otra posible explicación sería que los errores de clasificación de la diabetes, de la EA o de ambas hubieran atenuado la relación entre las dos enfermedades. En resumen, los resultados de este estudio coinciden con los de estudios longitudinales anteriores en los que se observó una débil asociación entre la diabetes y la EA, y una fuerte asociación entre la diabetes y la demencia asociada a ACV. Además, se extendieron estos resultados a grupos minoritarios de la población y se comprobó que el riesgo poblacional de demencia asociada a ACV atribuible a la diabetes es significativamente mayor en los negros y los hispanoamericanos que en los blancos. (Luchsinger JA, Tang M-X, Stern Y, Shea S, Mayeux R. Diabetes mellitus and risk of Alzheimer's disease and dementia with stroke in a multiethnic cohort. Am J Epidemiol 2001;154:635-641) 